•综述・

\title{
探讨监测传粉者的方法
}

\author{
童泽宇 ${ }^{1} \quad$ 徐环李 $^{2}$ 黄双全 $^{1^{*}}$ \\ 1 (华中师范大学生命科学学院进化与生态学研究所, 武汉 430079) \\ 2 (中国农业大学植保学院昆虫学系, 北京 100193)
}

\begin{abstract}
摘要: 种子植物的有性生殖依赖于花粉传递, 传粉者是花粉传递的媒介。传粉者为野生植物和农作物提供的传粉 服务, 是我们绿色星球最重要的生态过程之一, 在维持生物多样性和农作物生产方面具有重要作用。农业集约化、 生境破碎、全球气候变暖等因素加剧了传粉者衰减和灭绝的风险, 对生态系统的功能和农业生产造成了不利影响。 为了维系植物与传粉者生态互作关系的稳定性, 人们建立了一系列从局部地区到国际、由普通民众到科研人员参 与的传粉者监测项目, 以期掌握传粉生态系统的状况和发展趋势, 为自然和农业生态系统的健康提供预警和反 馈。本文强调了监测传粉者的首要前提条件, 即正确区分传粉者和访花者; 总结了监测传粉者的直接和间接方法, 包括群落水平的直接观察监测, 以及利用关联数据进行间接推断与调查; 介绍了具有潜力的由大众参与的公众监 测项目。针对 7 种常见传粉者类群, 讨论了可行的适用于各类群的监测方法, 为监测拓展到更多的传粉者类群提供 了可能。期望能为生物多样性的保护、传粉者动态的精准监测提供建议与参考。
\end{abstract}

关键词：传粉者; 传粉者监测; 生物保护; 生物多样性; 植物-传粉者相互作用

\section{Examining methodologies of pollinator detection in the field}

\author{
Zeyu Tong ${ }^{1}$, Huanli Xu², Shuangquan Huang ${ }^{2 *}$ \\ 1 Institute of Evolution and Ecology, School of Life Sciences, Central China Normal University, Wuhan 430079 \\ 2 Department of Entomology, College of Plant Protection, China Agricultural University, Beijing 100193
}

\begin{abstract}
Sexual reproduction of seed plants depends largely on pollen transfer. The pollination service provided by pollinators for wild plants and managed crops is one of the most crucial ecological processes on our planet, as it plays an essential role in sustaining biodiversity and crop production. Factors such as agricultural intensification, habitat fragmentation, and global climate change have increased the risk of pollinator decline and extinction, which would have detrimental effects on ecological function and agricultural production. To maintain the stability of ecological interactions between plants and pollinators, a series of pollinator monitoring schemes have been established, ranging from the regional to international scale. Participants including volunteer citizens and professional scientists have obtained the status and trends of pollination systems, thereby helping to provide early alerts and feedbacks for the risk of natural and agricultural ecological systems. In this view examining the methodologies of pollinator monitoring, we emphasize that it is necessary to distinguish pollinators from floral visitors. A diversity of direct and indirect methods for monitoring pollinators is summarized for seven types of animals (including Lepidoptera, Coleoptera, Hymenoptera, Diptera, Aves, Mammalia, and Lacertilia, respectively). A simple monitoring program that includes volunteer participation is also recommended. Commonly used field monitoring strategies for seven groups of pollinators would be useful as references for monitoring additional pollinator faunas. The pros and cons of these diverse methods for protecting and monitoring pollinators are discussed, which is useful for the long-term detection of pollinator dynamics.
\end{abstract}

Key words: pollinator; pollinator monitoring; biological protection; biodiversity; plant-pollinator interactions 
植物是生态系统的奠基者, 通过光合作用将太 阳能转化为植物自身和其他生物可以利用的化学 能。据估计, 现生植物中被子植物(或称有花植物) 最为繁盛, 约有295,383种 (Christenhusz \& Byng, 2016)。由于固着生长的习性, 被子植物的有性生殖 依赖一定的传粉媒介, 将花粉(包裹着精子)从植物 的雄性结构(花药)传递到䧳性结构(䧳荵, 包裹着卵 细胞), 从而实现受精、结实并产生后代。除一些植 物利用风或水作为花粉传递媒介外, 地球上约 87.5\%的被子植物物种由动物传粉(Ollerton et al, 2011)。传粉者为植物提供的生态服务保障了大多数 野生植物的繁殖, 因而对整个生态系统的健康十分 关键(Allen-Wardell, 1998; Fontaine et al, 2006)。在农 林生态系统中, 传粉者的多寡和传粉服务的质量对 那些依赖动物传粉的作物的产量和质量至关重要, 全球作物产量的35\%依赖于动物传粉者(Klein et al, 2007)。据估计传粉者为大约75\%的作物物种提供重 要的生态服务(Kleijn et al, 2015), 2009年全球依赖 传粉者的作物的经济价值高达 3,610 亿美元 (Lautenbach et al, 2012)。

当前, 全球范围内出现了传粉者衰减的趋势, 这将对传粉服务的质量、生态系统的功能以及人类 的食物和健康安全带来不利影响(Vanbergen \& the Insect Pollinators Initiative, 2013; Potts et al, 2016), 这些影响已经逐渐受到人们的关注(Cunningham, 2000; Committee on the Status of Pollinators in North America, 2007; IPBES, 2016)。目前世界范围内已经 实施了众多地区级、国家级以及全球级别的传粉者 监测方案和计划, 参与人员包括科学家和专业人员 以及受训的志愿者。但现有监测方案中仍存在一些 问题, 如对传粉者的监测偏向于蜂类, 而非蜂类传 粉者提供的传粉服务对许多野生植物和农作物同 样重要(Larson et al, 2001; Rader et al, 2016); 监测 项目往往忽略尚不濒危的传粉者类群, 如蝇类; 监 测目标注重传粉者本身而忽略传粉者与植物之间 建立的联系; 采用被动采样法的监测系统无法区分 访花者和真正的传粉者。在实际调查中, 要避免将 花上的动物都记录为传粉者, 如害虫、捕食者、寄 生者(图1a)、盗粉者(图1b)等。另外, 传粉者监测系 统的风险预警和反馈功能也需要改善。随着人们对 传粉者重要性认识的进一步加深, 未来对传粉者的 监测将会逐步完善, 以利于保护、维持生态系统的
健康和农业生产的发展。

\section{访花者不一定是传粉者}

传粉 (pollination) 是花粉从植物的雄性生殖器 官传递到植物雌性生殖器官的过程。传粉者 (pollinator) 是一类与植物发生相互作用的动物, 在 这一过程中充当花粉传递的载体(Huang \& Guo, 2000)。访花者(floral visitor)是访问花的动物, 这些 动物出于各自的目的, 如采集或盗取花的报酬、啃 食花结构、利用花作为产卵地，甚至只是偶尔停留。 显然访花者不一定是传粉者，只有当访花者将某植 物的花粉传递到同种植物的柱头上时，才能称之为 该植物的传粉者。

一种植物可以拥有许多种来自不同类群的传 粉者。因身体结构特性和行为模式的不同，不同传 粉者的有效程度也有所不同。例如在有些植物中, 频繁出现的访花者传粉效率反而低于较少出现的 传粉者(Wilson \& Thomson, 1991)。衡量不同传粉者 有效性的一个指标是花粉传递效率，即落置到柱头 上的花粉占传粉者移出花粉的比例(Johnson et al, 2005)。在单次访问中，传粉者移出更少花粉的同时 将更多花粉落置到柱头上，即可达到更高的花粉传 递效率。虽然许多访花者在某种植物上是有效或高 效的传粉者, 但在另一些植物上可能是低效传粉者, 甚至无法起到传粉作用。

监测传粉者的重要工作之一是确认真正的传 粉者(Willmer, 2011)。确定真正的传粉者可以为监测 项目提供更为精确的监测目标，从而节省人力和财 力并产出更有意义的数据。为了确定某种动物为传 粉者, 需要: (1)确认该种动物是访花者。观察设立 的样方或随机选择的花序、拍摄记录访花者的行为 并采集鉴定访花者的种类。(2)确认该种访花者与花 有足够多的有效互动。观察访花者在访问时身体结 构是否能匹配花的结构, 植物的花药和柱头是否能 直接或间接地触碰到传粉者(图1b)，访花者在访问 过程中是否主动收集花粉或被动承载花粉(图1c), 并成功地将花粉传递到柱头上。蜂类传粉者在主动 收集花粉时会通过梳理行为将花粉转移至腿部的 花粉筐，这样落置在传粉者身体上的花粉不容易与 柱头接触而难以实现传粉(图1d)。通过鉴定蜂类身 体和腿部花粉笚中花粉的种类也可判断其是哪些 植物的传粉者, 不过需要注意这些花粉笚中的花粉 

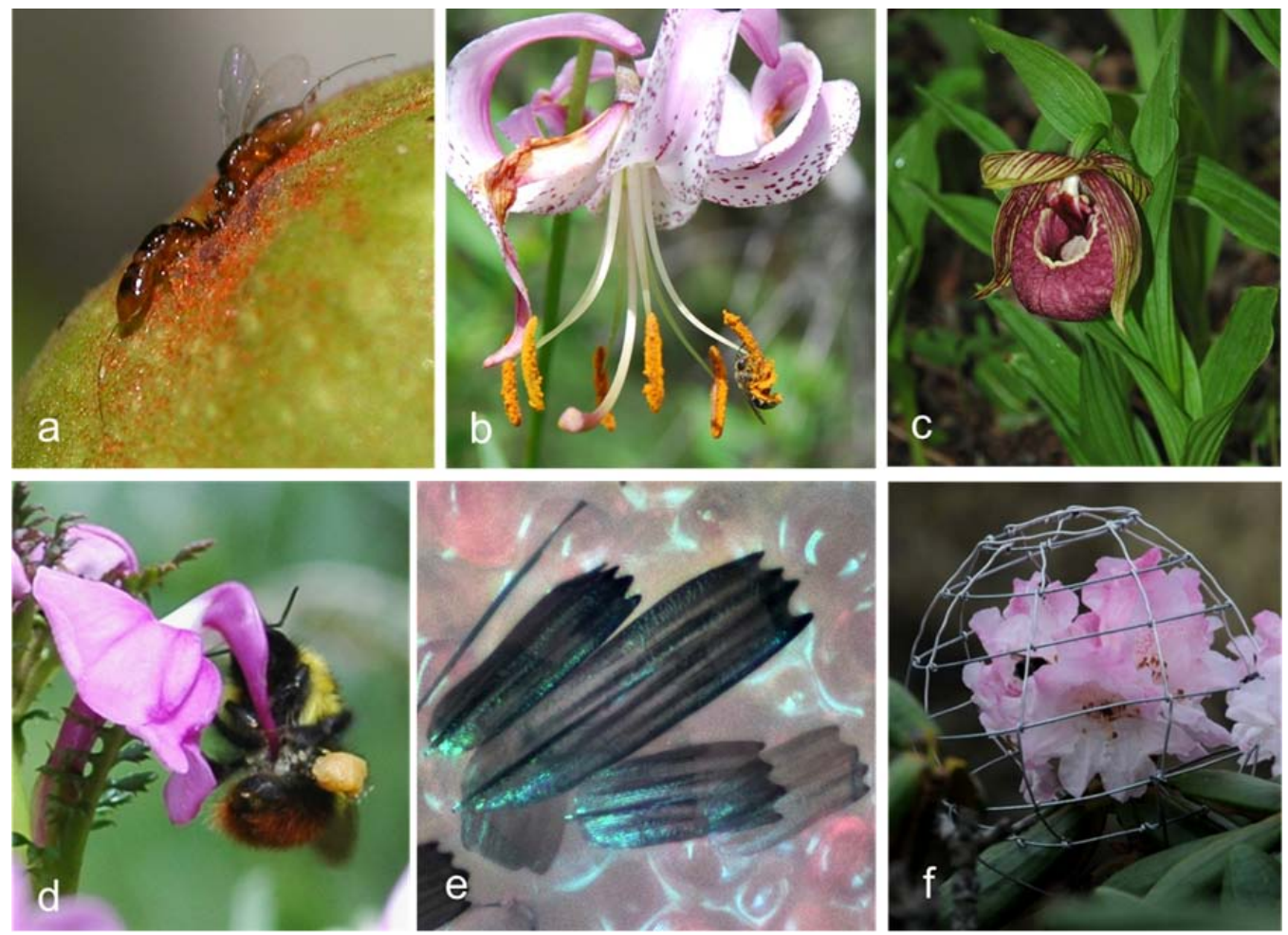

图1 访花者的行为及为推断其是否为传粉者而采取的不同实验方法。(a)两只欺骗性的小蜂(Eupristina sp.)试图进入高山榕 (Ficus altissima)的隐头花序，这类蜂是非传粉小蜂。(b)一种淡脉隧蜂(Lasioglossum sp.)采集宝兴百合(Lilium duchartrei)的花 粉, 由于虫体大小远小于花部雌雄器官之间的距离, 这类隧蜂是盗粉者, 虫体不接触柱头而不起传粉作用。(c)为检验何种昆 虫可能是西藏杓兰(Cypripedium tibeticum)的传粉者，实验者抓捕不同体型的昆虫放入囊状的下唇中，如果爬出的昆虫能带 走西藏构兰的花粉块, 则推断其为可能的有效传粉者。(d)红腹熊蜂(Bombus friseanus)在四川头花马先蒿(Pedicularis cephalanta) 上收集花粉, 注意柱头接触虫体的部位; 被熊蜂收集到花粉筐中的花粉不能用于传粉。(e)图片显示的是落置在柳兰 (Chamerion angustifolium)柱头上的蛾类昆虫鳞片, 通过显微镜观察柱头是否有鳞片落置, 可以间接推断夜间的蛾类访 花。(f)为检验麻点杜鹃(Rhododendron clementinae)是否有鸟类传粉者, 可将金属丝制成的笼子安置在花序外，隔离鸟类访花 但允许昆虫访问, 注意照片中有一只熊蜂在访花。

Fig. 1 Photo illustrations of behaviors of floral visitors and different methods for detecting potential pollinators. (a) Two female cheaters (Eupristina sp.) are trying to enter the hypanthium of Ficus altissima, these are non-pollinating agaonid fig wasps. (b) A halictid bee (Lasioglossum sp.) is collecting pollen from Lilium duchartrei. It acts as a pollen robber rather than a pollinator because its body size is too small to mechanically fit the distance between the male and female organs. (c) To investigate the potential pollinators for Cypripedium tibeticum, investigators let captured insects with different body sizes get into the inflated labellum, if the escaped insects could carry the pollinia of this orchid then the visitor could be likely an effective pollinator. (d) A bumblebee (Bombus friseanus) is collecting pollen from Pedicularis cephalantha. Note the stigma contact position of the pollinator body. Those pollen grains collected by the bee into the corbiculae are no longer useful for pollination. (e) Scales from a moth visitor deposited on a stigma of Chamerion angustifolium with microscopy. Nocturnal visits by moths could be indirectly inferred via checking scale deposition on stigmas. (f) To examine whether Rhododendron clementinae can be pollinated by birds, investigators used cages made by metal wires to cover the inflorescence, isolating bird visits but allowing insect visits. Note a bumblebee is visiting the caged inflorescence.

常常是多种花粉的混合, 有些花粉是该蜂类个体从 他种植物上收集的异种花粉(Fang \& Huang, 2016)。

植物的花通常会有适应于传粉者行为的特征, 分析这些特征可以排除一些偶尔访花而实际无传 粉功能的访花者。包括: (1)确定该种植物的繁育系 统是自交还是异交。一些植物可以通过自动自交或 传粉者促进的自交而授粉(Lloyd \& Schoen, 1992), 这种情况下的访花者其传粉贡献可能甚微。验证植
物自身是否需要传粉服务，可以通过对比隔离/不 隔离传粉者时的坐果率、结籽率来确定。(2)确认该 种植物的花期、每日开放时间是否与访花者的活跃 时间相匹配; 调查该种植物的花部报酬类型、相应 访花者对报酬的需求以及这些报酬呈现的时间是 否响应访花者的活动规律。例如在西双版纳分布的 大戟科植物黄蓉花(Dalechampia bidentata), 其有效 传粉者条切叶蜂(Megachile faceta)在花中采集树脂 
用于筑巢(Armbruster et al, 2011)。

如何监测传粉者

\section{1 群落水平直接观察监测}

自然群落中, 往往多种开花植物与多种传粉者 构成了互作网络(方强和黄双全, 2014)。监测单一物 种难以全面获知传粉者的访花行为信息, 而群落水 平上的监测则可获取多种植物与多种传粉者的物 种状态以及双方的互作关系, 并用于构建生态互作 网络。目前依托动植物互作网络进行的生态监测都 力求考察种间互作的模式和强度 (Kaiser-Bunbury et al, 2010; Menz et al, 2011)。生态互作网络不仅提 供群落结构的数据, 还可提供生态系统功能方面的 评估(Gibson et al, 2006; Bascompte \& Jordano, 2007), 因而可用作衡量生态恢复的指标。例如 KaiserBunbury等(2017)分析了塞舌尔群岛8个样地64个群 落的植物-传粉者网络, 通过对比植被恢复和未恢 复群落中 10 种多度最高的植物的生殖表现, 得出生 态系统的恢复可以显著提升传粉者物种数量、访问 次数和互作多样性, 且恢复群落里的植物-传粉者 互作关系比未恢复群落更为泛化、具有更高的功能 性生态圥余。

样线法(transect)是监测植物-传粉者互作的一 种有效方法(Westphal et al, 2008)。例如Hegland等 (2010)设置3条50 m长的样线, 在2003-2004年的5-8 月, 每3天从08:00-17:00在这些样线上行走调查, 记 录遇到的访花者种类及其访问的植物种类。设置固 定样方也是一种常用方法, 例如 Fang 和 Huang (2016)在 12 个 $1 \mathrm{~m} \times 1 \mathrm{~m}$ 的观测样方中观察了 14 种植 物与65种传粉者的相互作用, 并构建了传粉者在植 物种间与种内的访问网络和植物的异种与本种花 粉传递网络; 在监测过程中, 观察时间的分配基于 单个植物物种, 使每个物种观察时间均等, 避免因 观察者偏好导致数据偏差。

监测传粉网络涉及到众多植物与传粉者物种 以及它们之间的连接关系, 所以随着监测尺度增大, 人力和财力消耗也会剧增。为了提高监测的成本效 率(cost-efficiency), 可将监测时间集中于植物-传粉 者互作的高峰时期, 或将监测目标集中于网络中重 要的植物与传粉者类群。例如Hegland等(2010)报道, 如果将监测时间集中于植物-传粉者互作的高峰时 期, 那么以约 $20 \%$ 的时间和经费投入即可获取传粉
网络中前20种重要传粉者70-85\%的生态功能信息。

\section{2 间接推断与调查}

间接推断与调查同样可以为传粉者的监测提 供可靠的数据支持。例如Aizen和Harder (2009)利用 联合国食物与农业组织(Food and Agriculture Organization of the United Nations, FAO)的数据分析指 出, 全球人工蜂箱数量在过去的半个世纪里增加了 约 $45 \%$, 而在同样的时间里全球农业对动物传粉服 务的需求增加了 $300 \%$ 以上。

如果传粉者与植物之间联系紧密, 那么定期调 查植物坐果率和结实率可用于粗略估计传粉者的 多度，当传粉者极为稀少或难以直接观察时，这种 方法可以作为常规监测方法的替代(Willmer, 2011)。 调查植物的多度与丰富度同样也可间接揭示其传 粉者的状况。Carvell等(2006)调查了分布于英国的 熊蜂食源植物, 发现其中 $76 \%$ 的植物物种在 20 世纪 晚期经历了衰减，从而也为熊蜂种群的衰减提供了 间接证据。

\section{3 大众参与的公众监测}

传粉者的时空分布不连续, 进行大尺度监测费 时费力(Roubik, 2001; Williams et al, 2001), 由科学 家和专业调查人员主导的监测项目时常固于人力 限制。公民科学(citizen science)中的公众监测项目 可充分发挥大众的力量, 突破时间、空间和人力的 限制。在公众监测项目中，参与者经过适当的训练 即可收集到可靠的传粉者观察数据(Altizer et al, 2004; Kremen et al, 2011)。公众监测项目成本低廉, 所需经费投入较低，例如在欧洲，据估计参与公民 科学的志愿者每年可节省大约 400 万欧元的科研经 费(Schmeller et al, 2009)。公众监测项目还具有可观 的社会价值，为民众提供一定程度的教育和娱乐 (Bonney et al, 2009)。当然, 公众监测项目也存在一 些问题, 如数据质量难以控制、稀有物种难以兼顾 等(Genet \& Sargent, 2003)。

针对传粉者的公众监测项目应制定明确的监 测目标, 建立一套标准化的数据采样方法和简便易 学的志愿者培训教程, 并采用标准化的校正方案提 高数据质量。例如Roy等(2016)开展了一年期公众监 测项目“Big Bumblebee Discovery”，调查了英国各 地不同种类的熊蜂访问多种薰衣草(Lavendula spp.) 的情况。志愿者是约1.3万名7-11岁的小学生，根据 项目制定者设计的简洁、通用的方式调查一定时间 
内在随机地点熊蜂访问薰衣草的次数, 使用项目发 放的简易检索表鉴定熊蜂种类, 并将数据上传至数 据库以备分析。

目前国内已有可通过上传照片帮助鉴定植物 的手机应用, 如花伴侣、形色、微软识花等, 如果 未来有类似的昆虫鉴定网络平台, 将大大提高公众 参与的热情和监测质量。

\section{4 针对不同传粉者类群的监测方法}

因不同类群传粉者的习性和生境各不相同, 监 测难度和最适监测方法各异。根据监测目的、经费 预算、时间消耗和人力成本, 监测方法按精度可分 为3个层次：(1)低精度的方法仅监测可作为传粉者 的动物类群的多样性指标, 因而在利用传粉生物学 手段确认传粉者身份后, 常规的针对各类动物的监 测方法可以直接加以应用, 所得数据可用于评估传 粉类群宏观变动的趋势; (2)一般精度的方法用于监 测那些出现在有花植物附近的访花者和潜在的访 花者类群, 可以间接获得动物与植物相互作用的联 系, 但需要注意这种联系通常弱于真实情况; (3)高 精度要求观察到的访花者必须都是经过隔离实验 验证的有效传粉者, 需要图片或录像以及动物标本 作为凭证, 并尽可能地记录传粉者与植物之间的直 接相互作用，如访花者和植物的种类、访问次数、 访问频率等，以便构建生态互作网络。

\subsection{1 鳞翅目昆虫传粉者的监测}

据估计鳞翅目昆虫中的传粉者种数最多, 约有 141,600 种, 占所有传粉者种数的 $40.53 \%$, 其中仅蛾 类(异角亚目) 就有 123,100种 (35.24\%) (Ollerton, 2017)。有分析显示一些鳞翅目昆虫中的蛾类和蝴蝶 的种群数量锐减, 其原因主要是栖息地丧失和气候 的变化(Merckx et al, 2013)。

蛾类提供重要的传粉服务, 但常被人们忽视, 重要原因之一是蛾类通常夜间活动(Philipp et al, 2006)。夜行蛾类的活跃时间通常是18:30-22:00, 在 观察时可使用红色塑料片或红布盖住手电筒, 或使 用红外手电筒提供照明以降低对蛾类的干扰(Liu \& Huang, 2013; Xiong et al, 2015); 带有微光摄录功能 的摄像机可以辅助摄录傍晚至日落前的蛾类行为; 使用红外夜视仪或带有夜视功能的摄像机则可以 直接在约 $30 \mathrm{~cm}$ 的距离上清晰观察、记录蛾类的访 问行为。

监测一些白天活动的蛾类可以直接采用样线
法。例如Kutt等(2016)监测澳大利亚的一种日行的 濒危金阳蛾(Synemon plana)时设立了 90 个 $100 \mathrm{~m}$ 长 的样线, 在监测对象最为活跃的时期(南半球的 11 月至翌年1月)展开调查。在每个天气适宜的调查日, 监测从上午 10 点开始, 持续到下午 2 点。调查者在样 线上缓步行走，每间隔 $5 \mathrm{~m}$ 搜索、计数一次附近的蛾 类并记录主要的环境因子，如每半个小时的云层情 况、风速和温度等。

翅膀上的鳞片是鳞翅目昆虫的一大特性。在确 定某种植物仅由蛾类传粉的情况下, 可以通过检查 花上落置的鳞片数量间接推断蛾类的访问情况(图 1e)。例如Rodger等(2013)以一种台湾百合 (Lilium formosanumz) 柱头上监测到的甘薯天蛾 (Agrius convolvuli)鳞片数量作为该类传粉者的访问指标。 鳞片的残留物可在野外直接用 20 倍的手持式放大 镜检测并统计。但由于这是一种间接方法，最好结 合照片、花粉落置等来提供多方印证。

与蛾类相比, 蝶类常在白天活动而为人们所熟 知。因为对生境和气候改变十分敏感，蝶类被提议 作为生物多样性的指示类群(Thomas, 2005)。国际上 已有针对蝴蝶的长期监测计划，如在英国有始于 1976年的传统蝴蝶监测计划 (traditional Butterfly Monitoring Scheme, tBMS), 主要针对半自然状态 的蝶类栖息地。在每年的 4 月 1 日至9月 29 日蝴蝶成 体活跃时期，研究人员在一天中的特定时刻沿着固 定的样线 (全长平均为 $1.9 \mathrm{~km}$ ) 行走调查, 每周至少 一次(Roy et al, 2015)。另一项监测计划是更广地区 蝴蝶调查(Wider Country Butterfly Survey, WCBS), 位于英国乡野各处的监测地区被划分为大量 $1 \mathrm{~km}^{2}$ 的方格, 每个方格内设立两条平行的 $1 \mathrm{~km}$ 样线, 参 与者被随机分配于不同的方格区域内，于7-8月之 间在样线中行走调查两次(Brereton et al, 2011)。

\subsection{2 鞘翅目昆虫传粉者的监测}

可作为传粉者的鞘翅目昆虫约有 77,300 种，占 所有传粉者种数的 $22.13 \%$, 是物种数量第二大的传 粉者类群(Ollerton, 2017)。虽然鞘翅目昆虫种类众 多，但其体表光滑不易携带花粉，且通常不会多次 连续访花, 因而常常只能作为一种泛化的传粉者 (龚燕兵和黄双全, 2007; Johnson et al, 2007)。

对鞘翅目昆虫的监测方法主要分为陷阱法和 目视法(visual encounter survey, VES) (Chiari et al, 2013)。陷阱法包括用于采集步行甲虫的巴氏诱罐 
(pitfall trap, PT) 和用于采集飞行甲虫的飞行拦截陷 阱(aerial flight interception traps, AFIT) (Harvey et al, 2011)。巴氏诱罐的主体是埋入地下的瓶子或罐子, 容器的开口与地表平齐或略具向下的坡度, 利用地 表昆虫的活动习性, 捕获落入诱杯中的昆虫(周红 章等, 2014)。为了防止捕食者的干扰和雨水的影响, 可在诱罐上方加装顶棚; 为了提高捕获效率还可在 陷阱中装载诱饵。飞行拦截陷阱的典型代表是马氏 网(Malaise trap), 其主体结构如同一顶悬挂的蚊帐, 引导被拦截的昆虫通过顶部的凹槽进入收集管(吴 琼等, 2016)。陷阱的布设同样遵循样线法, 例如 Brooks等(2012)在监测遍布英国不同生境类型的12 个陆地样地上的步行甲虫时, 在每个样地设置了 3 条样线, 在每条样线以 $10 \mathrm{~m}$ 的间隔设立 10 个巴氏诱 罐, 在步行甲虫活跃时期每两周更新一次陷阱, 鉴 定并计数所有捕获的步行甲虫物种。在目视法的调 查中，步行甲虫可以用手直接抓取，飞行甲虫可以 用昆虫网捕获, 捕获后的甲虫在标记后放回, 根据 再次捕获的频率可以估算甲虫的多度和丰富度。

许多情况下目视法更适合于对鞘翅目昆虫的 监测。许多甲虫传粉的植物会在夜间闭合花朵 $(\mathrm{Li} \&$ Huang, 2009), 或形成较封闭的花部结构迫使甲虫 虫体被动携带花粉; 访花甲虫的传粉行为需要打开 花冠才可看到。Chiari等(2014)在对甲虫的监测中对 比了陷阱法和目视调查法的效果, 发现虽然陷阱法 的人力和时间消耗更少, 但即便装载陷阱, 其捕获 效果也有限; 由于一些甲虫种类的雌雄个体习性不 同, 导致陷阱法捕获的甲虫具有强烈的性别偏差; 陷阱容易受到捕食者的攻击。相对而言, 目视法获 取的信息更为全面、准确, 具有更高的成本效率。

\subsection{3 膜翅目昆虫传粉者的监测}

据估计膜翅目昆虫约有70,000种可作为传粉者, 占所有传粉者种数的 $20.04 \%$ (Ollerton, 2017), 其中 蜂类被认为是世界范围内最为重要的传粉者类群 (Klein et al, 2007; Potts et al, 2016)。我国已被命名的 传粉蜂有 1,000 多种, 但对这些传粉蜂的种群数量、 空间分布特征、受威胁因素等知之甚少(徐环李等, 2009)。

有多种采样方法可用于对膜翅目昆虫的监测。 Westphal等(2008)对比了6种广泛使用的针对膜翅目 蜂类昆虫的采样方法: (1)定点样方。设立固定大小 的样方, 在设定时长内记录样方内所有访花蜂类。 (2)标准化样线行走调查(standard transect walks)。设 立固定长度的样线，观查者沿样线以设定的时间行 走, 记录所有观察到的访花物种。(3)多变样线行走 调查(variable transect walks)。在1 ha的样地中, 调查 者在随机选定的资源丰富的植物斑块中，以固定的 周期观察并采集蜂类。(4)盘诱法(pan trap)。盘诱法 是一种陷阱法，主体是一个盛水的盘子，通常向盘 内加一滴洗洁精降低水的表面张力, 使昆虫更易沉 没。(5)用芦苇管制作的巢陷阱(trap nest)。该方法诱 使洞巢蜂类进入芦苇管制作的人工巢中产卵，待调 查结束后将孵化的成体制成标本并鉴定到种。(6) 用纸管制作的巢陷阱。通过这6种采样方法的对比, Westphal等(2008)认为盘诱法是对蜂类而言最为通 用、适合的监测方法, 因为此方法采样效率高、不 受观察者的经验影响。如果监测目的是为了兼顾到 洞巢蜂类, 则应考虑用巢陷阱; 如果调查者经验丰 富, 且需要开展更为详细的针对植物 - 传粉者相互 作用的研究, 则样线法更为适宜。

Lebuhn等(2013)比较分析了7种常见监测蜂类 的采样方法, 同样认为如果综合考虑年际变异系数 的波动、花费和难度，盘诱法是最适合用于监测蜂 类多度和丰富度变化的采样方法。此外, 他们还估 算监测传粉蜜蜂群落变动需要抽样调查200-250个 地点, 一年采样 2 次, 持续 5 年以上的数据才能正确 评估传粉者每年2-5\%物种丰富度的下降。

\subsection{4 双翅目昆虫传粉者的监测}

据估计双翅目昆虫传粉者约有55,000种，占所 有传粉者物种的 $15.74 \%$ (Ollerton, 2017), 主要包括 食蚜蝇科和蜂虹科昆虫。

人们尚未充分重视双翅目昆虫的传粉价值, 因 而对传粉者衰减的担忧还没有扩展到双翅目昆虫 传粉者(Biesmeijer et al, 2006), 许多已有的监测项 目和方法将双翅目昆虫视作害虫(如Hamby et al, 2014)。实际上双翅目蝇类昆虫可作为至少555种有 花植物的传粉者或经常性的访花者，且可以为超过 100 种栽培植物传粉，包括可可、芒果、腰果、茶和 洋苾等(Larson et al, 2001)。一些植物类群开花散发 出难闻的气味以吸引蝇类昆虫为其传粉。除了蝇类, 蚊子、瘞蚊等都被观察到给植物(例如马淣铃属 Aristolochia、五味子属Schisandra等)传粉。在一些 情况下双翅目昆虫传粉者的重要性甚至超过蜂类 传粉者。例如在北极和高山区域蜂类传粉者缺乏, 
占主导地位的双翅目昆虫为当地植物贡献了主要 的传粉服务(Kearns \& Inouye, 1994; Elberling \& Olesen, 1999)。双翅目昆虫传粉者不仅容易受到天 气因素影响, 如起雾、阴天、刮风和下雨等(Inouye et al, 2015), 也容易受到气候变化的影响, 因为气 候变化可直接影响双翅目昆虫的活跃程度, 也可通 过影响食源植物花期而造成间接影响(Iler et al, 2013)。可见造成其他传粉者类群衰减的因素同样也 可对双翅目昆虫带来影响。

对双翅目昆虫的监测开展较早, 但没有确定它 们是否为传粉者。例如Whittaker (1952)对美国田纳 西和北卡莱罗纳州的Great Smoky山脉的双翅目昆 虫开展了广泛的监测, 采样方法是在 16 个样地的众 多样点进行相同次数(50次)的扫网，一共采集并鉴 定了8,000只双翅目昆虫。Siemann等(1996)在1992 年美国明尼苏达州雪松溪的 48 个样地对双翅目昆 虫进行调查, 在每个样地使用相同次数(100次)的扫 网采样，总共收集并鉴定了1,167种昆虫的89,596份 标本。

\subsection{5 鸟类传粉者的监测}

据估计可作为传粉者的鸟类有 1,089 种 (Ollerton, 2017)。鸟类传粉对许多植物的生殖和生存至关 重要(Allen-Wardell, 1998), 为植物-传粉者互作生 态系统的功能性冗余做出了重要贡献(Aizen \& Feinsinger, 1994)。

区域性的鸟类调查方法较为成熟。例如梁健超 等(2017)使用样线法调查鸟类, 将调查区域划分为 若干个 $10 \mathrm{~km} \times 10 \mathrm{~km}$ 的网格, 每个网格依据具体情 况设置1条调查样线, 每条样线调查3次。调查时以 望远镜结合鸣声加以辨别, 记录样线上遇到的鸟类 种类、个体数量、栖息生境和海拔高度。高歌等(2017) 使用红外相机监测鸟类, 利用GIS在地形图上设置 若干 $100 \mathrm{~m} \times 100 \mathrm{~m}$ 的网格, 在每个网格内选择鸟类 容易出现的区域布设红外相机，累计监测了10,400 台日，用拍摄到的照片和视频鉴定物种名称并用作 分析多样性和时间变化。

具有代表性的跨地区乌类监测项目是始于 1966年的北美繁育鸟类调查(North American Breeding Bird Survey, BBS), 该项目主要由志愿者参与, 现已成为北美鸟类居群变化动态的首要信息来源 (Sauer et al, 2013)。项目由众多观察路径组成, 每条 观察路径每年至少由一位观察者检测一次, 在每条
观察路径上选取间距 $800 \mathrm{~m}$ 的 50 个独立观察点。受 训的观察者在每个观察点通过肉眼观察和声音听 辨来鉴定鸟类, 在 $3 \mathrm{~min}$ 的观察期内记录半径 $400 \mathrm{~m}$ 内的所有鸟类。

如要重点监测作为传粉者的鸟类, 则需通过隔 离授粉实验来验证鸟类访花的有效性(图1f)。例如 Sun等(2017)在研究访花鸟类与金花茶(Camellia petelotii)之间的相互作用时，使用细眼纱布网隔绝 所有传粉者来判断植物是否能自花授粉，用金属丝 制成小鸟笼安置在花序周围以隔绝鸟类但允许昆 虫访问，从而判断鸟类传粉的重要性。同时考察了 安置鸟笼本身是否影响了昆虫的访问, 记录了昆虫 分别在有、无鸟笼情况下的访问频率。结果表明隔 绝鸟类不影响昆虫访问，但导致金花茶的结实率和 结籽率大幅降低; 此外测量所得该植物的花蜜特征 (大量的、稀释的花蜜常为鸟类所偏好), 也为鸟类传 粉提供了间接证据。

因为鸟类访问的植物常为高大木本，且鸟类生 性敏捷，容易觉察到周围环境的异动，给监测带来 一定难度。Fang等(2012)使用长焦相机远距离观察刺 探花蜜或触碰花药的鸟类个体; 同时考虑到鸟类常 常成群行动, 所以在观察时, 随机选取一只鸟计数 其访花次数, 随后用访花次数乘以这群鸟的数量再 除以花序上的总花数，作为该时间段内的访问频率。

\subsection{6 哺乳动物传粉者的监测}

据估计作为传粉者的哺乳动物有 344 种, 其中 翼手目 236 种，其他非飞行哺乳动物有 108 种 (Ollerton, 2017)。这里主要介绍针对翼手目和啮齿 目动物的监测。

世界上许多地区都分布有可提供传粉服务的 蝙蝠。与常见传粉者类群相似, 蝙蝠与植物也能建 立高度互惠的联系, 如长舌食蜜蝙蝠(Anoura fistulata)可将舌头伸出口外 $8.5 \mathrm{~cm}$, 约为其体长的 1.5 倍, 而相应的一种桔梗科植物Centropogon nigricans 具 有8-9 $\mathrm{cm}$ 的长花冠, 只由该种蝙蝠传粉(Muchhala \& Thomson, 2009)。

蝙蝠多为夜间行动，且物种鉴定有难度，因而 对蝙蝠的监测具有一定挑战性。英国1996年建立了 国家蝙蝠监测计划(National Bat Monitoring Project, NBMP), 计划的主要参与者是大量受过基础培训的 志愿者。志愿者使用不同的方法在每年的不同时间 段进行调查, 旨在监测蝙蝠种群的动态变化, 为保 
护行动提供参考(Barlow et al, 2015)。此计划的基础 监测方法有4种: (1)栖木(roost)计数。在夏季(5-7月 之间)日落时分, 志愿者按照指导教程寻找栖木并 使用蝙蝠探测器调查。(2)冬眠调查。在冬季(1-2月, 也可放宽至12-3月)的山洞、矿井、地下室或冷库, 志愿者在调查地周围的路径搜索处于冬眠状态的 蝙蝠。(3)野外调查。志愿者被随机分配在 $1 \mathrm{~km}^{2}$ 的 地理方格内, 使用蝙蝠探测器沿着长约 $3 \mathrm{~km}$ 的样线 调查。(4)水道调查。主要针对沿水栖息、贴水飞行 的蝙蝠, 调查区域被预先划分为 $100 \mathrm{~m}^{2}$ 的地理方格, 志愿者沿着水道调查总长度 $1 \mathrm{~km}$ 的样线。

很早以来就有啮齿动物可以作为传粉者的报 道。例如Cocucci和Sérsic (1998)在花旁放置烟熏玻 璃板, 通过检查板上的足迹来间接推断夜行啮齿动 物的访花行为。又如Johnson等(2001)在南非的半干 旱性的Succulent Karoo地区发现风信子科植物艳镜 (Massonia depressa) 在夜间至少可被4种啮齿动物传 粉。花旁布设的陷阱所捕获的夜行啮齿动物, 其口 鼻部检测到有大量艳镜的花粉; 使用大孔径网袋罩 住植物排除啮齿动物访问而允许昆虫访问, 结果发 现只结了很少的种子; 通过对泌蜜规律的测量得知 该种植物报酬的分泌动态与夜行啮齿动物的活跃 时间相符, 花蜜中的果冻状内含物可防止昆虫盗取, 但适合啮齿动物硚食。这些直接证据和间接证据都 表明啮齿动物可以作为传粉者。

由于啮齿动物体型较小, 行踪不定, 所以对啮 齿动物传粉者的监测存在一定难度。视频监测技术 可用于该类群与植物间互作关系数据的收集和分 析(赵清建等, 2016)。例如Wester等(2009)发现夜行 啮齿动物纳马夸岩鼠 (Micaelamys namaquensis) 可 以访问风信子科植物二叶塔玉风(Whiteheadia biflolia)的花, Melidonis和Peter (2015) 确定了山龙眼科多 叶海神花 (Protea foliosa) 几乎只由日行的纹鼠 (Rhabdomys pumilio)提供传粉服务。在实地调查中, 监测相机架设在植物上方, 镜头对准较为完好的花 序, 并确保视野内至少存在一个已经开裂的花药。 现在大多数远程摄影设备已能提供很高的图像分 辨率以确定访花者种类、访问频率、植物的雌雄荵 与访花者身体接触的位置, 以及花粉落置于访花者 身体的部位(Zoller et al, 2016)。

\subsection{7 蜥蜴传粉者的监测}

据统计全世界有37种蚚蜴可作为传粉者(Oller- ton, 2017)。蜥蜴传粉常常发生在与大陆隔绝的海岛 上(Olesen \& Valido, 2003), 蚚蜴传粉者以花蜜为食, 在访花时身体特定部位(如喉部鳞片)可携带花粉 (Eifler, 1995)。蚚蜴传粉的植物也出现了一些适应性 特征, 例如在非洲东海岸的毛里求斯群岛, 当地的 锦葵科植物Trochetia sp.分泌红色或黄色花蜜以吸 引岛上的壁虎(Phelsuma sp.)为其传粉(Hansen et al, 2006), 而一般虫媒传粉植物的花蜜是无色透明的 液体。

针对蜊蜴的监测, Lettink和Monks (2016)总结 了3类方法: 第一类是主动的全面搜索。调查者分白 天和黑夜，在蜥蜴可能出没的任何地方检视、清查 出现的蚚蜴个体以及行踪指示物，如蜕皮和粪便 等。这种方法可以获得最为全面的信息, 但人力消 耗较大。第二类是被动的陷阱捕捉，包括设置巴氏 诱罐来捕捉非树栖蚚蜴、设置漏斗陷阱来捕捉树栖 蜊蜴并定期统计捕获物。这种方法适用于长期监测, 但需要预先制作并设置陷阱。与陷阱原理类似的方 法还有制作人工避难所诱使蚚蜴入住并统计，不过 这类人工避难所的设立需要在调查前 $1-3$ 个月完 成。第三类是使用照片比对。通过对监测区域拍摄 高分辨率照片, 并将照片与参照物对比, 将带有特 定视觉标记的蜥蜴个体区分出来。尽管这一方法存 在精度限制, 但避免了监测过程中与蚚蜴个体的直 接接触, 适合应用于小种群的濒危物种。

\section{结语}

完善传粉者监测方法离不开传粉生物学研究 的理论和基础，而传粉生物学研究的进步依赖于创 造及利用各式各样的新方法(Fægri \& van der Pijl, 1979; Kearns \& Inouye, 1993)。为了应对传粉者衰减 所带来的一系列生态系统功能危机, 人们已经开展 了一系列跨地区的传粉者监测项目，这些监测项目 应用了多种多样的监测方法。

归因于传粉者行为的多变性和类群的特异性, 单一的监测方法无法兼顾到监测的各个层面。使用 多种监测方法可以实现数据和信息的互补，与此同 时，如果能够区分传粉者、明确监测目标，则可以 更加科学有效地选择主要的监测方法。依赖于各类 摄录技术的进步、依托数据共享平台实现的监测数 据网络化和信息化, 以及新的数据分析方法的提出, 未来的传粉者监测方法将会更加趋于完善, 使得监 
测数据更易获取、精确度和可信度更高、人力资源 和经济投入的成本效率更高、覆盖的传粉者类群更 加全面, 为传粉者的衰减趋势提供及时的预警, 为 决策者制定保护传粉者的策略提供参考。

致谢：感谢中国科学院西双版纳热带植物园彭艳 琼研究员提供榕小蜂照片, 熊英泽、黄至欢博士和 博士研究生宋云澎提供部分实验处理照片。感谢 华中师范大学传粉与进化实验室研究生参与有益 的讨论。

\section{参考文献}

Aizen MA, Feinsinger P (1994) Habitat fragmentation, native insect pollinators, and feral honey bees in Argentine "Chaco Serrado”. Ecological Applications, 4, 378-392.

Aizen MA, Harder LD (2009) The global stock of domesticated honey bees is growing slower than agricultural demand for pollination. Current Biology, 19, 915-918.

Allen-Wardell G (1998) The potential consequences of pollinator declines on the conservation of biodiversity and stability of food crop yields. Conservation Biology, 12, 8-17.

Altizer S, Hochachka WM, Dhondt AA (2004) Seasonal dynamics of mycoplasmal conjunctivitis in eastern North American house finches. Journal of Animal Ecology, 73, 309-322.

Armbruster WS, Gong YB, Huang SQ (2011) Are pollination "syndromes" predictive? Asian Dalechampia fit neotropical models. The American Naturalist, 178, 135-143.

Barlow KE, Briggs PA, Haysom KA, Hutson AM, Lechiara NL, Racey PA, Walsh AL, Langton SD (2015) Citizen science reveals trends in bat populations: The National Bat Monitoring Programme in Great Britain. Biological Conservation, 182, 14-26.

Bascompte J, Jordano P (2007) Plant-animal mutualistic networks: The architecture of biodiversity. Annual Review of Ecology, Evolution, and Systematics, 38, 567-593.

Biesmeijer JC, Roberts SP, Reemer M, Ohlemüller R, Edwards M, Peeters T, Schaffers AP, Potts SG, Kleukers R, Thomas CD, Settele J, Kunin WE (2006) Parallel declines in pollinators and insect-pollinated plants in Britain and the Netherlands. Science, 313, 351-354.

Bonney R, Cooper CB, Dickinson J, Kelling S, Phillips T, Rosenberg KV, Shirk J (2009) Citizen science: A developing tool for expanding science knowledge and scientific literacy. BioScience, 59, 977-984.

Brereton TM, Cruickshanks KL, Risely K, Noble DG, Roy DB (2011) Developing and launching a wider countryside butterfly survey across the United Kingdom. Journal of Insect Conservation, 15, 279-290.
Brooks DR, Bater JE, Clark SJ, Monteith DT, Andrews C, Corbett SJ, Beaumont DA, Chapman JW (2012) Large carabid beetle declines in a United Kingdom monitoring network increases evidence for a widespread loss in insect biodiversity. Journal of Applied Ecology, 49, 1009-1019.

Carvell C, Roy DB, Smart SM, Pywell RF, Preston CD, Goulson D (2006) Declines in forage availability for bumblebees at a national scale. Biological Conservation, 132, 481-489.

Chiari S, Zauli A, Mazziotta A, Luiselli L, Audisio P, Carpaneto GM (2013) Surveying an endangered saproxylic beetle, Osmoderma eremita, in Mediterranean woodlands: A comparison between different capture methods. Journal of Insect Conservation, 17, 171-181.

Chiari S, Zauli A, Audisio P, Campanaro A, Donzelli PF, Romiti F, Svensson GP, Tini M, Carpaneto GM (2014) Monitoring presence, abundance and survival probability of the stag beetle, Lucanus cervus, using visual and odourbased capture methods: Implications for conservation. Journal of Insect Conservation, 18, 99-109.

Christenhusz MJ, Byng JW (2016) The number of known plants species in the world and its annual increase. Phytotaxa, 261, 201-217.

Committee on the Status of Pollinators in North America (2007) Status of pollinators in North America. National Academies Press, Washington, DC.

Cocucci AA, Sérsic AN (1998) Evidence of rodent pollination in Cajophora coronata (Loasaceae). Plant Systematics and Evolution, 211, 113-128.

Cunningham SA (2000) Depressed pollination in habitat fragments causes low fruit set. Proceedings of the Royal Society B: Biological Sciences, 267, 1149-1152.

Eifler DA (1995) Patterns of plant visitation by nectar-feeding lizards. Oecologia, 101, 228-233.

Elberling H, Olesen JM (1999) The structure of a high latitude plant-flower visitor system: The dominance of flies. Ecography, 22, 314-323.

Fang Q, Chen YZ, Huang SQ (2012) Generalist passerine pollination of a winter-flowering fruit tree in central China. Annals of Botany, 109, 379-384.

Fang Q, Huang SQ (2014) Progress in pollination ecology at the community level. Chinese Science Bulletin, 59, 449-458. (in Chinese with English abstract) [方强, 黄双全 (2014) 群落水平上传粉生态学的研究进展. 科学通报, 59, 449-458.]

Fang Q, Huang SQ (2016) A paradoxical mismatch between interspecific pollinator moves and heterospecific pollen receipt in a natural community. Ecology, 97, 1970-1978.

Fægri K, van der Pijl L (1979) The Principles of Pollination Ecology, 3rd edn. Pergamon Press, Oxford.

Fontaine C, Dajoz I, Meriguet J, Loreau M (2006) Functional diversity of plant-pollinator interaction webs enhances the persistence of plant communities. PLoS Biology, 4, 129-135. 
Gao G, Wang B, He CX, Luo X (2017) Biodiversity of birds and mammals in alpine habitat of Mt. Gaoligong, Lushui County, Yunnan. Biodiversity Science, 25, 332-339. (in Chinese with English abstract) [高歌, 王斌, 何臣相, 罗旭 (2017) 云南沪水高黎贡山高山生境的鸟兽多样性. 生物 多样性, 25, 332-339.]

Genet KS, Sargent LG (2003) Evaluation of methods and data quality from a volunteer-based amphibian call survey. Wildlife Society Bulletin, 31, 703-714.

Gibson RH, Nelson IL, Hopkins GW, Hamlett BJ, Memmott J (2006) Pollinator webs, plant communities and the conservation of rare plants: Arable weeds as a case study. Journal of Applied Ecology, 43, 246-257.

Gong YB, Huang SQ (2007) On methodology of foraging behavior of pollinating insects. Biodiversity Science, 15, 576-583. (in Chinese with English abstract) [龚燕兵, 黄双 全 (2007) 传粉昆虫行为的研究方法探讨. 生物多样性, 15, 576-583.]

Hamby KA, Bolda MP, Sheehan ME, Zalom FG (2014) Seasonal monitoring for Drosophila suzukii (Diptera: Drosophilidae) in California commercial raspberries. Environmental Entomology, 43, 1008-1018.

Hansen DM, Beer K, Müller CB (2006) Mauritian coloured nectar no longer a mystery: A visual signal for lizard pollinators. Biology Letters, 2, 165-168.

Harvey DJ, Hawes CJ, Gange AC, Finch P, Chesmore D, Farr I (2011) Development of non-invasive monitoring for larvae and adults of the stag beetle, Lucanus cervus. Insect Conservation and Diversity, 4, 4-14.

Hegland SJ, Dunne J, Nielsen A, Memmott J (2010) How to monitor ecological communities cost-efficiently: The example of plant-pollinator networks. Biological Conservation, 143, 2092-2101.

Huang SQ, Guo YH (2000) New advances in pollination biology and studies in China. Chinese Science Bulletin, 45, 1441-1447.

Iler AM, Inouye DW, Hoye TT, Miller-Rushing AJ, Burkle LA, Johnston EB (2013) Maintenance of temporal synchrony between syrphid flies and floral resources despite differential phenological responses to climate. Global Change Biology, 19, 2348-2359.

Inouye DW, Larson BM, Ssymank A, Kevan PG (2015) Flies and flowers III: Ecology of foraging and pollination. Journal of Pollination Ecology, 16, 115-133.

IPBES (2016) Summary for policymakers of the assessment report of the Intergovernmental Science-Policy Platform on Biodiversity and Ecosystem Services on pollinators, pollination and food production. In: Intergovernmental Science-Policy Platform on Biodiversity and Ecosystem Services Deliverables of the 2014-2018 Work Programme (eds Potts SG, Imperatriz-Fonseca VL, Ngo HT, Biesmeijer JC, Breeze TD, Dicks LV, Garibaldi LA, Hill R, Settele J, Vanbergen AJ, Aizen MA, Cunningham SA, Eardley C,
Freitas BM, Gallai N, Kevan PG, Kovács-Hostyánszk A, Kwapong PK, Li J, Li X, Martins DJ, Nates-Parra G, Pettis JS, Rader R, Viana BF), pp. 1-28. IPBES, Bonn, Germany.

Johnson SD, Ellis A, Dötterl S (2007) Specialization for pollination by beetles and wasps: The role of lollipop hairs and fragrance in Satyrium microrrhynchum (Orchidaceae). American Journal of Botany, 94, 47-55.

Johnson SD, Neal PR, Harder LD (2005) Pollen fates and the limits on male reproductive success in an orchid population. Biological Journal of the Linnean Society, 86, 175-190.

Johnson SD, Pauw A, Midgley J (2001) Rodent pollination in the African lily Massonia depressa (Hyacinthaceae). American Journal of Botany, 88, 1768-1773.

Kaiser-Bunbury CN, Traveset A, Hansen DM (2010) Conservation and restoration of plant-animal mutualisms on oceanic islands. Perspectives in Plant Ecology, Evolution and Systematics, 12, 131-143.

Kaiser-Bunbury CN, Mougal J, Whittington AE, Valentin T, Gabriel R, Olesen JM, Blüthgen N (2017) Ecosystem restoration strengthens pollination network resilience and function. Nature, 542, 223-227.

Kearns CA, Inouye DW (1993) Techniques for Pollination Biologists. University Press of Colorado, Louisville.

Kearns CA, Inouye DW (1994) Fly pollination of Linum lewisii (Linaceae). American Journal of Botany, 8, 1091-1095.

Klein AM, Vaissière BJ, Cane H, Steffan-Dewenter I, Cunningham SA, Kremen C, Tscharntke T (2007) Importance of pollinators in changing landscapes for world crops. Proceedings of the Royal Society of London B: Biological Sciences, 274, 303-313.

Kleijn D, Winfree R, Bartomeus I, Carvalheiro LG, Henry M, Isaacs R, Klein AM, Kremen C, M'Gonigle LK, Rader R, Ricketts TH, Williams NM, Adamson NL, Ascher JS, Báldi A, Batáry P, Benjamin F, Biesmeijer JC, Blitzer EJ, Bommarco R, Brand MR, Bretagnolle V, Button L, Cariveau DP, Chifflet R, Colville JF, Danforth BN, Elle E, Garratt MPD, Herzog F, Holzschuh A, Howlett BG, Jauker F, Jha S, Knop E, Krewenka KM, Féon VL, Mandelik Y, May EA, Park MG, Pisanty G, Reemer M, Riedinger V, Rollin O, Rundlöf M, Sardiñas HS, Scheper J, Sciligo AR, Smith HG, Steffan-Dewenter I, Thorp R, Tscharntke T, Verhulst J, Viana BF, Vaissière BE, Veldtman R, Ward KL, Westphal C, Potts SG (2015) Delivery of crop pollination services is an insufficient argument for wild pollinator conservation. Nature Communications, 6, 7414.

Kremen C, Ullmann KS, Thorp RW (2011) Evaluating the quality of citizen-scientist data on pollinator communities. Conservation Biology, 25, 607-617.

Kutt AS, Dalton K, Wills TJ (2016) Identification of reliable predictors of golden sun moth Synemon plana habitat over multiple survey years can benefit conservation, restoration and surveys for new populations. Journal of Insect Conservation, 20, 691-699. 
Larson BMH, Kevan PG, Inouye DW (2001) Flies and flowers: Taxonomic diversity of anthophiles and pollinators. Canadian Entomologist, 133, 439-465.

Lautenbach S, Seppelt R, Liebscher J, Dormann CF (2012) Spatial and temporal trends of global pollination benefit. PLoS ONE, 7, e35954.

Lebuhn G, Droege S, Connor EF, Gemmill-Herren B, Potts SG, Minckley RL, Griswold T, Jean R, Kula E, Roubik DW, Cane J, Wright KW, Frankie G, Parker F (2013) Detecting insect pollinator declines on regional and global scales. Conservation Biology, 27, 113-120.

Lettink M, Monks JM (2016) Survey and monitoring methods for New Zealand lizards. Journal of the Royal Society of New Zealand, 46, 16-28.

Li JK, Huang SQ (2009) Effective pollinators of Asian sacred lotus (Nelumbo nucifera): Contemporary pollinators may not reflect the historical pollination syndrome. Annals of Botany, 104, 845-851.

Liang JC, Ding ZF, Zhang CL, Hu HJ, Duo HR, Tang H (2017) Bird diversity spatial distribution patterns and hotspots in Maixiu Area of Sanjiangyuan National Nature Reserve, Qinghai Province. Biodiversity Science, 25, 294-303. (in Chinese with English abstract) [梁建超, 丁志锋, 张春兰, 胡慧建, 朵海瑞, 唐虹 (2017) 青海三江源国家级自然保 护区麦秀分区乌类多样性空间格局及热点区域研究. 生 物多样性, 25, 294-303.]

Liu CQ, Huang SQ (2013) Floral divergence, pollinator partitioning and the spatiotemporal pattern of plant-pollinator interactions in three sympatric Adenophora species. Oecologia, 173, 1411-1423.

Lloyd DG, Schoen DJ (1992) Self-fertilization and cross-fertilization in plants. I. Functional dimensions. International Journal of Plant Sciences, 153, 358-369.

Melidonis CA, Peter CI (2015) Diurnal pollination, primarily by a single species of rodent, documented in Protea foliosa using modified camera traps. South African Journal of Botany, 97, 9-15.

Menz MH, Phillips RD, Winfree R, Kremen C, Aizen MA, Johnson SD, Dixon KW (2011) Reconnecting plants and pollinators: Challenges in the restoration of pollination mutualisms. Trends in Plant Science, 16, 4-12.

Merckx T, Huertas B, Basset Y, Thomas J (2013) A global perspective on conserving butterflies and moths and their habitats. In: Key Topics in Conservation Biology 2 (eds Macdonald DW, Willis KJ), pp. 237-257. John Wiley and Sons, Oxford.

Muchhala N, Thomson JD (2009) Going to great lengths: Selection for long corolla tubes in an extremely specialized bat-flower mutualism. Proceedings of the Royal Society of London B: Biological Sciences, 276, 2147-2152.

Olesen JM, Valido A (2003) Lizards as pollinators and seed dispersers: An island phenomenon. Trends in Ecology and Evolution, 18, 177-181.
Ollerton J (2017) Pollinator diversity: Distribution, ecological function, and conservation. Annual Review of Ecology, Evolution, and Systematics, 48, 353-376.

Ollerton J, Winfree R, Tarrant S (2011) How many flowering plants are pollinated by animals? Oikos, 120, 321-326.

Philipp M, Böcher J, Siegismund HR, Nielsen LR (2006) Structure of a plant-pollinator network on a pahoehoe lava desert of the Galápagos Islands. Ecography, 29, 531-540.

Potts SG, Imperatriz-Fonseca V, Ngo HT, Aizen MA, Biesmeijer JC, Breeze TD, Dicks LV, Garibaldi LA, Hill R, Settele J, Vanbergen AJ (2016) Safeguarding pollinators and their values to human well-being. Nature, 540, 220-229.

Rader R, Bartomeus I, Garibaldi LA, Garratt MPD, Howlett BG, Rachael W, Cunningham SA, Mayfield MM, Arthur AD, Andersson GKS, Bommarco R, Brittain C, Carvalheiro LG, Chacoff NP, Entling MH, Foully B, Freitas BM, Gemmill-Herren B, Ghazoul J, Griffin SR, Gross CL, Herbertsson L, Herzog F, Hipólito J, Jaggar S, Jauker F, Klein AM, Kleijn D, Krishnan S, Lemos CQ, Lindström SAM, Mandelik Y, Monteiro VM, Nelson W, Nilsson L, Pattemore DE, Pereira NDO, Pisanty G, Potts SG, Reemer M, Rundlöf M, Sheffield CS, Scheper J, Schüepp C, Smith HG, Stanley DA, Stout JC, Szentgyörgyi H, Taki H, Vergara CH, Viana BF, Woyciechowski M (2016) Non-bee insects are important contributors to global crop pollination. Proceedings of the National Academy of Sciences, USA, 113, 146-151.

Rodger JG, van Kleunen M, Johnson SD (2013) Pollinators, mates and Allee effects: The importance of self-pollination for fecundity in an invasive lily. Functional Ecology, 27, 1023-1033.

Roubik D (2001) Ups and downs in pollinator populations: When is there a decline? Conservation Ecology, 5, 2.

Roy DB, Ploquin EF, Randle Z, Risely K, Botham MS, Middlebrook I, Noble D, Cruickshanks K, Freeman SN, Brereton TM (2015) Comparison of trends in butterfly populations between monitoring schemes. Journal of Insect Conservation, 19, 313-324.

Roy HE, Baxter E, Saunders A, Pocock MJ (2016) Focal plant observations as a standardised method for pollinator monitoring: Opportunities and limitations for mass participation citizen science. PLoS ONE, 11, e0150794.

Sauer JR, Link WA, Fallon JE, Pardieck KL, Ziolkowski Jr DJ (2013) The North American breeding bird survey 1966-2011: Summary analysis and species accounts. North American Fauna, 79, 1-32.

Schmeller DS, Henry PY, Julliard R, Gruber B, Clobert J, Dziock F, Lengyel S, Nowicki P, Déri E, Budrys E, Kull T, Tali K, Bauch B, Settele J, van Swaay C, Kobler A, Babij V, Papastergiadou E, Henle K (2009) Advantages of volunteerbased biodiversity monitoring in Europe. Conservation Biology, 23, 307-316.

Siemann E, Tilman D, Haarstad J (1996) Insect species diversity, abundance and body size relationships. Nature, 380, 
704-706.

Sun SG, Huang ZH, Chen ZB, Huang SQ (2017) Nectar properties and the role of sunbirds as pollinators of the golden-flowered tea (Camellia petelotii). American Journal of Botany, 104, 468-476.

Thomas JA (2005) Monitoring change in the abundance and distribution of insects using butterflies and other indicator groups. Philosophical Transactions of the Royal Society of London B: Biological Sciences, 360, 339-357.

Vanbergen AJ, the Insect Pollinators Initiative (2013) Threats to an ecosystem service: Pressures on pollinators. Frontiers in Ecology and the Environment, 11, 251-259.

Wester P, Stanway R, Pauw A (2009) Mice pollinate the Pagoda lily, Whiteheadia bifolia (Hyacinthaceae): First field observations with photographic documentation of rodent pollination in South Africa. South African Journal of Botany, 75, 713-719.

Westphal C, Bommarco R, Carré G, Lamborn E, Morison N, Petanidou T, Potts SG, Roberts SPM, Szentgyörgyi H, Tscheulin T, Vaissière BE, Woyciechowski M, Biesmeijer JC, Kunin WE, Settele J, Steffan-Dewenter I (2008) Measuring bee biodiversity in different European habitats and biogeographical regions. Ecological Monographs, 78, 653-671.

Whittaker RH (1952) A study of summer foliage insect communities in the Great Smoky Mountains. Ecological Monographs, 22, 1-43.

Williams NM, Minckley RL, Silveira FA (2001) Variation in native bee faunas and its implications for detecting community changes. Conservation Ecology, 5, 7.

Willmer P (2011) Pollination and Floral Ecology. Princeton University Press, Princeton, New Jersey.

Wilson P, Thomson JD (1991) Heterogeneity among floral visitors leads to discordance between removal and deposition of pollen. Ecology, 72, 1503-1507.
Wu Q, van Achterberg C, Chen XX (2016) An introduction to the types of Malaise traps and their application for collecting insects. Chinese Journal of Applied Entomology, 53, 660-667. (in Chinese with English abstract) [吴琼, van Achterberg C, 陈学新 (2016) 昆虫诱集装置一马氏网 的类型与使用. 应用昆虫学报, 53, 660-667.]

Xiong YZ, Liu CQ, Huang SQ (2015) Mast fruiting in a hawkmoth-pollinated orchid Habenaria glaucifolia: An 8-year survey. Journal of Plant Ecology, 8, 136-141.

Xu HL, Yang JW, Sun JR (2009) Current status on the study of wild beepollinators and conservation strategies in China. Acta Phytophylacica Sinica, 36, 371-376. (in Chinese with English abstract) [徐环李, 杨俊伟, 孙洁茹 (2009) 我国 野生传粉蜂的研究现状与保护策略. 植物保护学报, 36, 371-376.]

Zhao QJ, Gu HF, Yan C, Cao K, Zhang ZB (2016) Impact of forest fragmentation on rodent-seed network. Acta Theriologica Sinica, 36, 15-23. (in Chinese with English abstract) [赵清建, 顾海峰, 严川, 曹科, 张知涁 (2016) 森林破碎化对鼠类-种子互作网络的影响. 兽类学报, 36, 15-23.]

Zhou HZ, Yu XD, Luo TH, Li XY, Wang FY, Li DE, Zhou YLZ, Zhao CY (2014) Collecting methods and sampling techniques of ground dwelling and predating Carabids and Staphylinids beetles. Chinese Journal of Applied Entomology, 51, 1367-1375. (in Chinese with English abstract) [周 红章, 于晓东, 罗天宏, 李晓燕, 王凤艳, 李德娥, 周毓 灵子，赵彩云 (2014) 土壤步甲和隐翅虫的采集与田间 调查取样技术. 应用昆虫学报, 51, 1367-1375.]

Zoeller KC, Steenhuisen SL, Johnson SD, Midgley JJ (2016) New evidence for mammal pollination of Protea species (Proteaceae) based on remote-camera analysis. Australian Journal of Botany, 64, 1-7.

(责任编委: 戈峰 责任编辑: 黄祥忠) 